\title{
Accurate Image Registration for Quadrature Tomographic Microscopy
}

\author{
Chia-Ling Tsai ${ }^{1}$, William Warger $\mathrm{II}^{2}$, and Charles DiMarzio ${ }^{2}$ \\ 1 National Chung Cheng University, Chiayi 62102, Taiwan \\ 2 Northeastern University, Boston, MA 02115, U.S.A
}

\begin{abstract}
This paper presents a robust and fully automated registration algorithm for registration of images of Quadrature Tomographic Microscopy (QTM), which is an optical interferometer. The need for registration of such images is to recognize distinguishing features of viable embryos to advance the technique for In Vitro Fertilization. QTM images a sample (live embryo) multiple times with different hardware configurations, each in turn producing 4 images taken by 4 CCD cameras simultaneously. Embryo movement is often present between imaging. Our algorithm handles camera calibration ${ }^{1}$ of multiple cameras using a variant of ICP, and elimination of embryo movement using a hybrid of feature- and intensity-based methods. The algorithm is tested on 20 live mouse embryos containing various cell numbers between 8 and 26. No failure thus far, and the average alignment error is 0.09 pixels, corresponding to the range of 639 and 675 nanometers.
\end{abstract}

\section{Introduction}

With the work pace and stress levels ever increasing, infertility now affects $10 \%$ [4] of the population at reproductive age. In Vitro Fertilization (IVF) is a method of assisted reproduction in which fertilization takes place in a laboratory dish. Usually, multiple embryos are transferred to the uterus during each cycle to increase the success rate. One cycle of IVF costs an average of $\$ 12,400$ in the United States. According to the latest statistics, the success rate for IVF is $29.4 \%$ live deliveries per egg retrieval, of which about $63 \%$ are singletons, $32 \%$ are twins, and $5 \%$ are triplets or more [4, 1,

To strive for a higher success rate of live deliveries and to reduce multiple births, researchers at Northeastern University, USA, are developing a unique state-of-the-art "fusion microscope" to recognize distinguishing features of viable embryos [6, 11. This instrument combines 5 imaging modalities for subsurface imaging, including Quadrature Tomographic Microscopy (QTM) and Differential Interference Contrast (DIC) microscopy.

There are two types of registration involved: inter- and intra-modality registration. The work presented in this paper is on intra-modality registration of

${ }^{1}$ In this paper, we define camera calibration as a process that estimates the spatial relationship between the 4 CCD cameras. 
QTM images. Applications for such work include the construction of amplitude and phase images of transparent samples, and cell counting methods involving the combination with DIC images for embryos before the blastocyst stage (less than 30 cells) [7.

\section{QTM Images and the Challenges}

QTM based on a modified Mach-Zener Interferometer. The laser source is split into two separate beams - signal and reference. The signal beam goes through the sample (embryo) and the reference beam travels the same distance as the signal beam, but is undisturbed. Both are combined later by an unpolarized beamsplitter and their interference is imaged simultaneously by 4 CCD cameras, each having its own coordinate system. An embryo is imaged three times by different hardware configurations (see Fig. 1): (a) mixture, the interference of the reference and signal beams, (b) reference only with the signal beam blocked, and (c) signal only with the reference beam blocked.

Placing all images in the same coordinate system requires calibration between the 4 cameras and registration of images taken at different times. The latter compensates for movement of the embryo. There are two ways to solve the registration problem: (a) for every image pair estimating the transformation directly from features of the embryo, and (b) solving camera calibration first and then eliminating embryo movement. The second method is superior in terms of speed and robustness for the following reasons:

- QTM images of all beam types appear substantially different (see Fig. 2). If registering such an image pair directly, the result is often not accurate
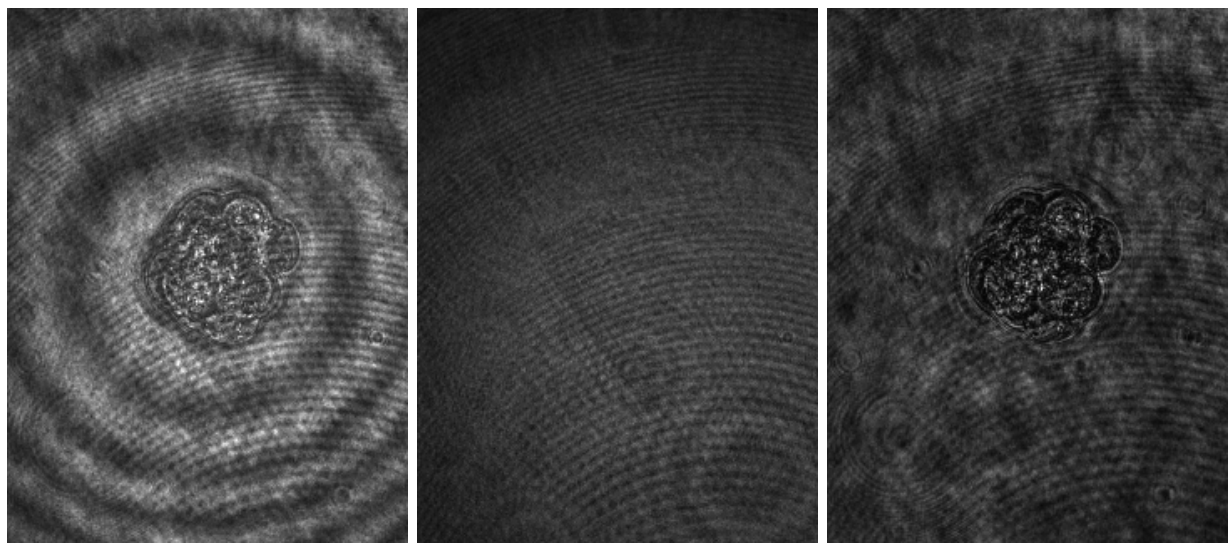

Fig. 1. QTM images of a live embryo taken with different hardware configurations. Accurate registration is required to eliminate the movement of the embryo between imaging. Left: mixture of reference and signal. Middle: reference alone. Right: signal alone. 

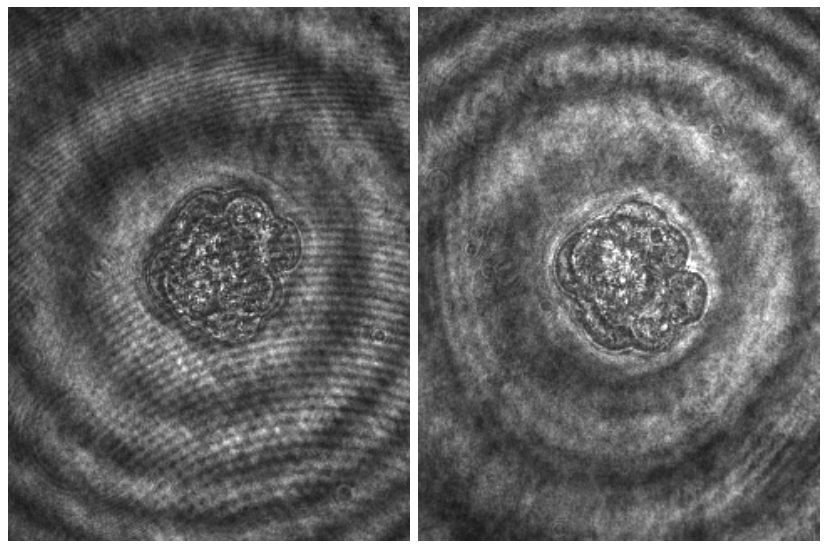

Fig. 2. Two mixture images taken by different cameras. Left: image of x-polarization (first camera). Right: image of y-polarization (second camera).

enough. In addition, it requires a more expensive registration algorithm that exploits the properties of the images. The number of times such an algorithm is applied should be minimized.

- Camera calibration is independent of the object for imaging and performed only when cameras change their relative positions. We can make use of any pattern that allows fast and accurate registration.

- If 4 images of a specific hardware configuration are aligned, we can make use of special optical properties to synthesize images that are similar in appearance. Such images can provide better registration result for elimination of embryo movement between imaging.

\section{Camera Calibration}

Between two images, the calibration process estimates an affine transformation that allows translation, rotation, reflection, and slight shearing. The image of the

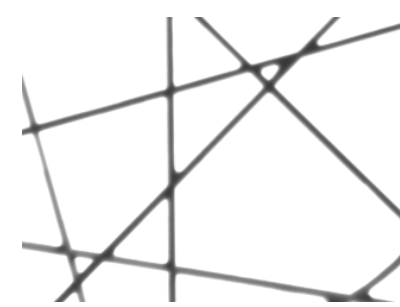

(a)

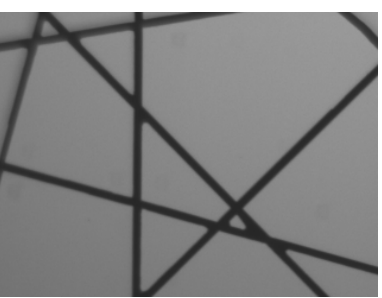

(b)

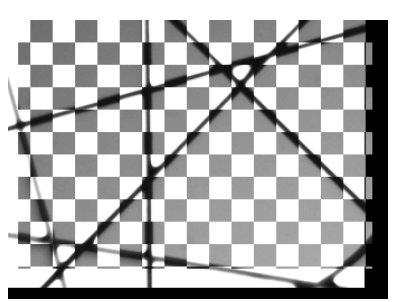

(c)

Fig. 3. The target pattern, imitating a vasculature, designed for automatic calibration of the 4 CCD cameras. (a) The image of the second camera. (b) The image of the fourth camera. (c) The checkerboard mosaic of the registered target images 
first camera is the anchor (fixed image) for registration. Since camera calibration is independent of the object for imaging, we designed a target pattern that simplifies the registration process. Images of the target are shown in Fig. 3 .

The pattern bears strong similarity with a vasculature - both are patterns of lines crossing at random angles. This property allows us to register our target images using feature-based methods in the literature. The features for registration are the centerline points and cross-overs of the dark lines [3, 12]. The transformation model is affine that allows left-right or top-bottom flipping, and significant rotation. We first discuss computation of the transformation $\Theta$ that does not involve reflection.

Estimation of $\Theta$ follows the method in [9], with some modifications, for full automation. The process consists of 2 steps:

Initialization: To avoid convergence to an incorrect alignment, we need a good initialization scheme to place the transformation in the right domain of convergence. We adopt invariant indexing to automate the process. In particular, for each image, a set of landmark (cross-over) pairs is generated and each pair is associated with a signature vector that is only invariant to similarity transformation, which is the initial transformation model. This is the reason for separation of reflection from the rest of the transformation. The algorithm generates a list of hypothesized correspondence pairs, ordered by the similarity of the invariant features. Each hypothesis provides an initial transformation.

Refinement: The refinement technique is a robust form of ICP (Iterative Closest Points) algorithm which minimizes the sum of point-to-line distances using an M-estimator. It alternates between closest centerline point matching using the current transformation, and transformation estimation using the set of matches. The transformation model for refinement is affine.

The algorithm refines the initial transformations one by one until it reaches one that converges to a good alignment. In practice, we only need to try the very first few.

Special care is needed to register an image pair related by reflection. To do so, the moving image is flipped by $\Theta_{f}$. Both the flipped and original moving images are registered to the fixed image using the abovementioned procedure, and the best alignment is taken. It does not matter if the moving image is right-left or top-bottom flipped, since rotation of $\pi$ relates one to the other. This is the major reason for the need of significant rotation. If the flipped is better aligned with the fixed image, the final transformation is the product of $\Theta$ and $\Theta_{f}$.

We validated the algorithm with a small set of target images. Fig. 3)(c) contains a checker-board mosaic with sub-pixel accuracy.

\section{Elimination of Embryo Movement}

The embryo may move between the time two images are acquired with different hardware configurations. In practice, the movement is limited to translation and 

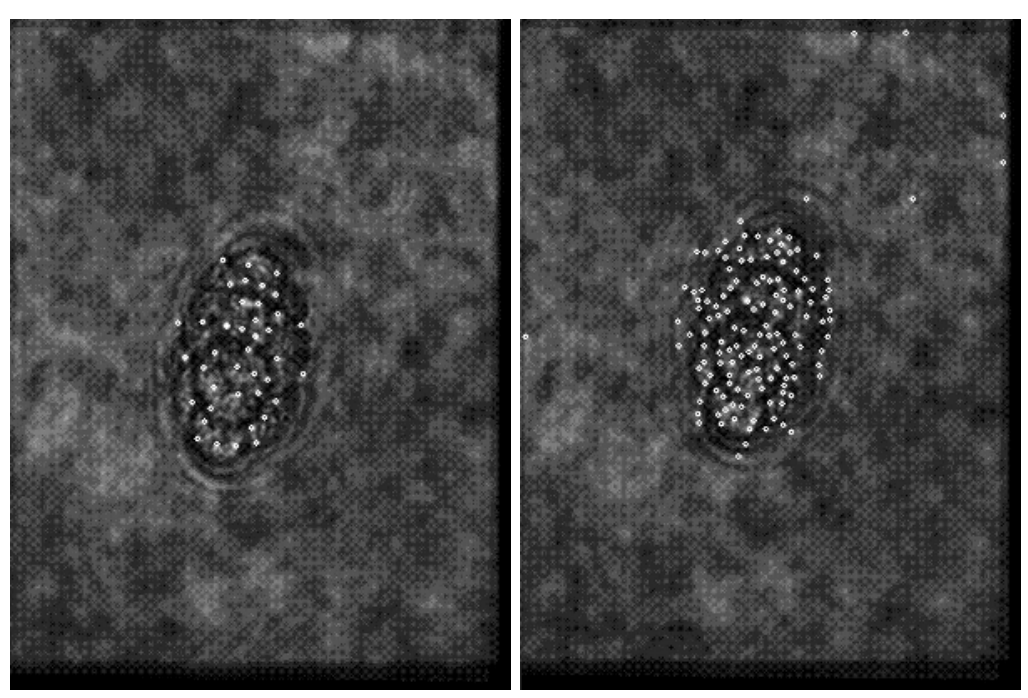

Fig. 4. The synthesized images for registration of signal to mixture images. Features, shown by white dots, are for invariant indexing and refinement. Left: Image $I_{a}$, average signal image. Only a subset of features extracted takes part in initialization and refinement. Right: Image $I_{s}$, the synthesized signal image. All features are involved in initialization.

rotation. To eliminate the movement, the signal images of the embryo must be aligned with the mixture. Due to the complex optical properties of the QTM, it is hard to directly relate the signal images to the mixture images (see Fig. 1). To overcome this problem, we perform the registration using synthesized images, instead of the original raw images.

Let $M_{i}$, be image $i$ of the mixture after camera calibration (i.e. the image mapped to the coordinate system of the first camera). Similarly, $R_{i}$ is for reference and $S_{i}$ for signal. The formula for the synthesized image $I_{s}$ is $\sum_{i=1}^{4}\left(M_{i}-R_{i}\right) / 4$. A very similar image $I_{a}$ can be generated from $S_{i}$ using the formula $\sum_{i=1}^{4} S_{i} / 4$.

$I_{a}$ is registered to $I_{s}$, which is in the same space as the first image of the mixture. Fig. 4 shows examples of $I_{a}$ and $I_{s}$ from the same set of images. The registration algorithm uses salient features and intensity structure of the images, and consists of 3 steps:

Feature Extraction: Since the background, not the embryo, is already aligned before registration, we want features that concentrate in the embryo to drive the registration out of the local minimum. Edge points are obviously not good candidates, since they are mostly on the fringes in the background. The type of key-point of our choice is harris-corner [5. We empirically determined the threshold on the corner-like measure to reduce the number of features extracted from the background. Fig. 4 shows a result of the feature extraction. 
Initialization: Robust initialization is crucial when the movement of the embryo is significant. Again, we employ invariant indexing. The invariant feature is Lowe's SIFT descriptor [8, a 128-component histogram of normalized gradients. The algorithm generates a list of hypothesized correspondence between two key-points, based on the invariant features. To reduce the number of hypotheses, we only take subset of key-points in $I_{a}$. The same set of keypoints are used in the refinement stage as well. Each hypothesis provides an initial affine transformation estimate from the location, orientation and scale of each key-point, and is refined separately.

Another common approach is to combine invariant features with RANSAC 2, which examines a number of $n$ correspondences ( $n$ is 3 for affine) for the best alignment. We chose invariant indexing over RANSAC for two reasons: (a) invariant indexing can return correct transformation as long as there is one correct match, and (b) SIFT descriptors are distinctive enough for this type of images that invariant indexing can succeed with the first match almost $100 \%$ of the time.

Refinement: The refinement algorithm is a variant of ICP that matches keypoints based on intensity structure [10]. Unlike initialization, which matches key-points detected in both $I_{a}$ and $I_{s}$, only key-points in $I_{a}$ are needed.

To generate the corresponding point in $I_{s}$, each key-point is associated with a small region $R$, centering at the location of the key-point $p$. This region is mapped into the other image $\left(I_{s}\right)$, using the current transformation estimate. Let the transformed location and region be $p^{\prime}$ and $R^{\prime}$, respectively. $R^{\prime}$ is matched against regions centered at pixel locations falling into the
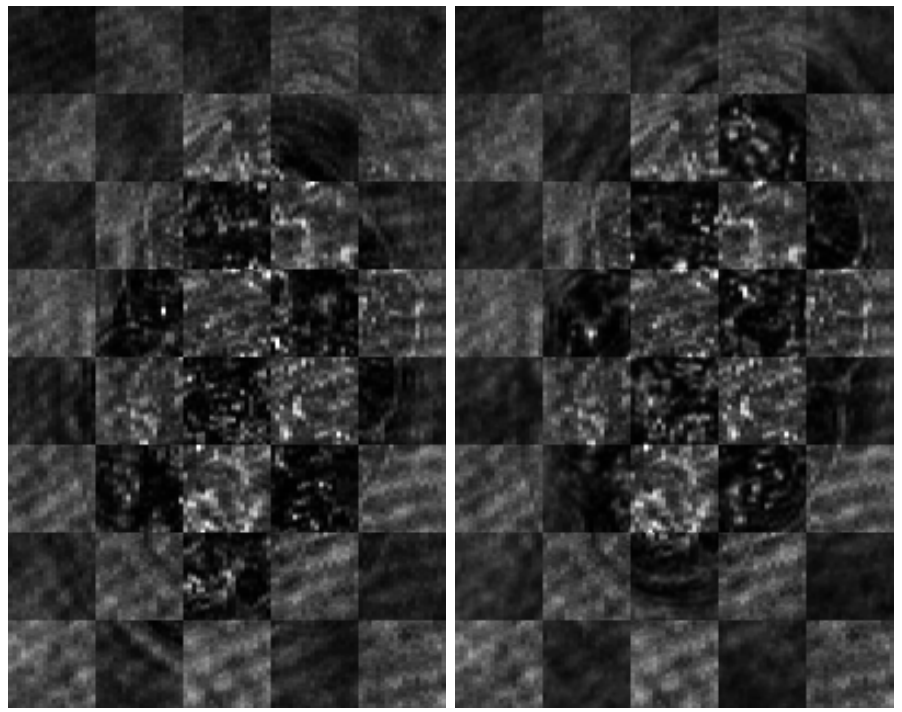

Fig. 5. The registration result, illustrated with cropped checker-board mosaics, of the average to synthesized signal images. Left: before registration. Discontinuity of the border of the embryo manifests the mis-alignment of the images. Right: after registration. 
search window of $p^{\prime}$ in $I_{s}$. The width of the window is a multiple of the error scale (uncertainty) of the current transformation; less accurate transformation leads to larger search window for each key-point. The best match has the lowest normalized SSD error measure. The center location of the best matched region defines the new corresponding point to $p$. Each match is assigned a robust weight which is a product of the distinctiveness of the match and the geometric distance between the corresponding points. The weights are required for robust estimation of the transformation.

We have tested the algorithm on a set of 20 mouse embryos containing various cell numbers between 8 and 26. Some samples were stationary, while others were fairly mobile. The success rate is $100 \%$ thus far, with an average alignment accuracy of 0.09 pixel, corresponding to 639 - 675 nanometers. The accuracy is measured in terms of weighted average distance error using the matches generated in the refinement stage. Fig. $[5$ is the registration result of the two images in Fig. 4. All results were verified manually by visual inspection of the checkerboard mosaics.

We have also attempted registration directly using signal and mixture images of the first camera. The results were not as satisfactory. The set of images in Fig. 1) 1 serves as an example of which signal and mixture images are substantially different. With this set of images, invariant indexing failed with the first 10 hypotheses, which was the threshold. Even if manually initialized by a pair of corresponding points, the final alignment was not as good as our current method.

\section{Discussion and Conclusion}

We presented a new algorithm for registration of QTM images, and demonstrated its robustness with images of a set of 20 live mouse embryos. The algorithm consists of two parts: camera calibration followed by registration of embryo images taken with different hardware configurations. After registration, all images are aligned with the first camera of mixture. If the embryo movement is present, the images of the embryo are aligned instead. The algorithm is both accurate and efficient - the average alignment error is 639-675 nanometers (0.09 pixel), and it succeeds with the first match almost all the time for both camera calibration and embryo registration.

With registration of QTM images in place, the next step is to apply the technique to synthesis of better amplitude and phase images, which are easily corrupted by embryo movement. Inter-modal registration of QTM phase images with DIC images, for applications such as cell counting, is also simplified by using the registered raw data (mixture and signal images) instead.

\section{References}

[1] A. Birmingham. Assisted reproductive technology in the united states: 2000 results generated from the american society for reproductive medicine/society for assisted reproductive technology registry. Fertility and Sterility, 81(5), May 2004. 
[2] M. Brown and D. Lowe. Recognising panoramas. In Proc. ICCV, 2003.

[3] A. Can, H. Shen, J. N. Turner, H. L. Tanenbaum, and B. Roysam. Rapid automated tracing and feature extraction from live high-resolution retinal fundus images using direct exploratory algorithms. IEEE Trans. on Inf. Tech. in Biomedicine, 3(2):125-138, 1999.

[4] A. S. for Reproductive Medicine. FACT SHEET: In vitro fertilization (IVF). http://www.asrm.org/Patients/FactSheets/invitro.html.

[5] C. Harris and M. Stephens. A combined corner and edge detector. pages 147-151.

[6] D. Hogenboom, C. DiMarzio, T. Gaudette, A. Devaney, and S. Lindberg. Threedimensional images generated by quadrature interferometry. Optics Letters, 23:783-785, 1998.

[7] W. W. II, J. Newmark, C. Chang, D. Brooks, C. Warner, and C. DiMarzio. Combining optical quadrature and differential interference contrast to facilitate embryonic cell counting with fluorescence imaging for confirmation. In Proc. SPIE Conference Imaging, Man. Ana. Bio. Cells, Tissue II, volume 5699, pages 334341, 2005.

[8] D. G. Lowe. Distinctive image features from scale-invariant keypoints. IJCV, 60(2):91-110, November 2004.

[9] C. Stewart, C.-L. Tsai, and B. Roysam. The dual-bootstrap iterative closest point algorithm with application to retinal image registration. IEEE Trans. Med. Imaging., 22(11):1379-1394, 2003.

[10] C. V. Stewart, Y.-L. Lee, and C.-L. Tsai. An uncertainty-driven hybrid of intensity-based and feature-based registration with application to retinal and lung CT images. In Proc. 7th MICCAI, Saint-Malo, France, 2004.

[11] D. Townsend, G. L. C. DiMarzio, and M. Rajadhyaksha. Multimodal optical microscope for imaging biological systems. In Proc. SPIE Conference 3D, Mult. Micro. Image Acqu. Pro. XII, volume 5701, pages 136-145, 2005.

[12] C.-L. Tsai, C. Stewart, B. Roysam, and H. Tanenbaum. Repeatable vascular landmark extraction from retinal fundus images using local vascular traces. IEEE Trans. on Inf. Tech. in Biomedicine, 8(2):122-130, 2004. 